TRANSACTIONS OF THE

AMERICAN MATHEMATICAL SOCIETY

Volume 353, Number 5, Pages 1741-1753

S 0002-9947(01)02420-5

Article electronically published on January 10, 2001

\title{
A FINITENESS THEOREM FOR HARMONIC MAPS INTO HILBERT GRASSMANNIANS
}

\author{
RODRIGO P. GOMEZ
}

This article is dedicated to my beloved daughter Katherine

\begin{abstract}
In this article we demonstrate that every harmonic map from a closed Riemannian manifold into a Hilbert Grassmannian has image contained within a finite-dimensional Grassmannian.
\end{abstract}

\section{INTRODUCTION}

Researchers such as Burns [3], Eells \& Wood [4], Uhlenbeck 11], and Atiyah [1] have investigated the properties of harmonic maps from Riemann surfaces into compact complex projective spaces as a so-called $\sigma$-model of the behavior of Yang-Mills theories in 4-dimensional manifolds. In particular, the latter two authors, as part of their investigations, considered mappings into loop groups (infinite-dimensional groups).

Our interest is in the behavior of harmonic maps from closed Riemann manifolds into Hilbert Grassmannians. Specifically, we wish to determine whether or not there exist any examples of harmonic maps which do not arise from the composition of harmonic maps into finite-dimensional Grassmannians with the inclusion of finitedimensional Grassmannians into the Hilbert Grassmannians. We will demonstrate that the answer is a resounding no.

Our proof will involve the space of solutions of a second-order linear elliptic differential equation of the sections of a Hilbert vector bundle. In order to make the proof easier to comprehend we will first give preliminary background material for our terms and properties. In Section 2 we define and state the relevant properties of our target manifolds, a Hilbert unitary Lie group $U_{H S}(H)$, a Banach unitary Lie group $U_{\text {res }}(H)$, as well as Hilbert vector bundles. In Section 3 we will define our Hilbert Grassmannians. We will exhibit a natural generalization of Cartan's embedding from Grassmannians into $U(n)$. In Section 4 we review briefly Hodge theory as it pertains to our computations. In Section 5 we will give a brief review of pseudo-differential operator theory and the necessary extension to Hilbert vector bundles which we will need. In Section [6we will define harmonic maps. In Section[7 we will at last state and prove our main theorems and corollary.

Remark. A closed manifold for us is a compact manifold which has no boundary.

Received by the editors May 22, 1997 and, in revised form, July 15, 1998.

2000 Mathematics Subject Classification. Primary 58E20; Secondary 53C07.

Key words and phrases. Harmonic maps, closed Riemannian manifolds.

I would like to thank D. Burns for suggesting this problem to me.

(C)2001 American Mathematical Society 


\section{Hilbert manifolds, Hilbert vector Bundles, ANd Lie Groups}

Remark. In this section and following sections $H$ will denote an infinite-dimensional separable complex Hilbert space. $S$ will denote the complex algebra of Hilbertschmidt operators acting on $H$.

Definition 2.1. A Hilbert manifold is just a manifold modelled on a Hilbert space $X$, in other words all local coordinate functions $f_{U}: U \subset M \rightarrow X$ map diffeomorphically onto a Hilbert space $X$. In a similar fashion we define a Banach manifold. A Hilbert vector bundle $V$ over a manifold $M$ is a vector bundle whose fibers are modelled on a Hilbert space $X$ and whose transitions functions take values in $G L(X)$. By a theorem of Kuiper, every Hilbert vector bundle $V$ over a compact manifold $M$ is trivial. It is trivial then to assign the constant metric to $V$ as is exhibiting a connection $\nabla$ on the space of sections $\Gamma(V)$. One simply starts with the flat connection and adds as a tensorial 1-form the differential of a function $f: M \rightarrow G L(X)$.

Since the theory of Lie groups is extensive (for example see 2] and [12]) we will limit ourselves to stating those properties of Lie groups which are directly relevant to our results.

Definition 2.2. Let $G L_{H S}(H)$ denote the Lie group of invertible operators of the form $I+x$ where $x \in S$.

To see that $G L_{H S}(H)$ is a group observe

1. for $I+x, I+y \in G L_{H S}(H)$, we have

$$
(I+x)(I+y)=I+(x+y+x y) .
$$

But $x+y+x y \in S$ so $(I+x)(I+y)$ is in $G L_{H S}(H)$.

2. Let $I+x \in G L_{H S}(H)$. Let $I+y:=(I+x)^{-1}$. To see that $y$ is in $S$ and hence that $I+y$ is in $G L_{H S}(H)$, notice that

$$
\begin{gathered}
I=(I+y)(I+x), \\
I=I+y+x+y x, \\
0=y+x+y x .
\end{gathered}
$$

Since $x, y x, 0$ are in $S$, then $y$ must be in $S$ as well. Hence $I+y=(I+x)^{-1}$ must be in $G L_{H S}(H)$.

It is a standard but lengthy argument to show $G L_{H S}(H)$ has the structure of a complex Hilbert manifold.

The Lie algebra $\mathcal{G} \mathcal{L}_{H S}(H)$ of $G L_{H S}(H)$ is simply $S$. The exponential map exp: $\mathcal{G} \mathcal{L}_{H S}(H) \rightarrow G L_{H S}(H)$ is the classical (holomorphic) map

$$
\exp (x):=I+x+\frac{1}{2 !} x^{2}+\cdots .
$$

The Lie algebra admits a bi-invariant Hermitian inner product defined by

$$
\langle x, y\rangle:=\operatorname{tr}\left(x^{*} y\right), \quad x, y \in \mathcal{G} \mathcal{L}_{H S}(H) .
$$

The associated (real) covariant derivative is given by

$$
\nabla_{x} y=\frac{1}{2}[x, y], \quad x, y \in \mathcal{G L}_{H S}(H) .
$$

As a consequence every 1-parameter subgroup $\exp (t x), x \in \mathcal{G} \mathcal{L}_{H G}(H)$, is a geodesic of $G L_{H S}(H)$ passing through $I$ and vice versa.

Now we consider a unitary subgroup of $G L_{H S}(H)$ : 
Definition 2.3. $U_{H S}(H)$ denotes the Lie subgroup of unitary operators of $G L_{H S}(H)$. Its Lie algebra $\mathcal{U}_{H S}(H)$ consists of the set of skew-Hermitian HilbertSchmidt operators. $\mathcal{U}_{H S}(H)$ has the advantage over the more general Lie algebra $\mathcal{U}(H)$, the space of bounded skew-Hermitian operators of $H$, that an inner product $\langle X, y\rangle:=\operatorname{tr}\left(X^{*} Y\right)$ can be defined for $\mathcal{U}_{H S}(H)$ whereas no inner product can be defined for $\mathcal{U}(H)$. Moreover, the inner product as defined for $\mathcal{U}_{H S}(H)$ is bi-invariant and the covariant derivative as defined in equation (7) makes sense for $\mathcal{U}_{H S}(H)$ as well. The exponential map exp: $\mathcal{U}_{H S}(H) \rightarrow U_{H S}(H)$ is a surjective map. To see this, consider an element $I+x \in U_{H S}(H)$. By comparing $(I+x)\left(I+x^{*}\right)$ and $\left(I+x^{*}\right)(I+x)$ we see that $x x^{*}=x^{*} x$.

It is well known that compact normal operators are diagonalizable. Hence, by a unitary transformation $U() U^{*}, I+x$ is similar to a diagonal matrix $d=$ $\operatorname{diag}\left(e^{i \theta_{1}}, \ldots, e^{i \theta_{j}}, \ldots\right)$. By a standard argument of taking the principal value of the logarithm of the entries and demonstrating that we arrive at a skew-Hermitian diagonal matrix $b$ whose entries form an $l_{2}$ sequence. Hence $I+x=\exp \left(U^{*} b U\right)$ and the proof is done.

Lastly, $U_{H S}(H)$ admits a canonical isometric involution $\sigma$ given by the map $\sigma(g)=g^{-1}$. Since $U_{H S}(H)$ is modelled upon $\mathcal{U}_{H S}(H)$ it is clear that $U_{H S}(H)$ is a Hilbert Lie group. As a manifold $U_{H S}(H)$ is thus a natural and well-behaved Lie group.

We define now the last Lie group that we require.

Definition 2.4. Let $H_{-}, H_{+}$be two closed, orthogonal, infinite-dimensional subspaces of $H$ such that they form a polar decomposition of $H$, i.e. $H=H_{+} \oplus H_{-}$. With respect to this decomposition, any operator $g \in L(H)$ can be decomposed into four maps

$$
\begin{aligned}
& g_{++}: H_{+} \rightarrow H_{+}, \quad g_{+-}: H_{+} \rightarrow H_{-}, \\
& g_{-+}: H_{-} \rightarrow H_{+}, \quad g_{--}: H_{-} \rightarrow H_{-} .
\end{aligned}
$$

We define as in [9] $U_{\text {res }}(H)$ as the group of unitary operators

$$
\left\{g \mid g_{++}, g_{--} \text {Fredholm, } g_{+-}, g_{-+} \text {Hilbert-Schmidt }\right\} \text {, }
$$

The Lie algebra $\mathcal{U}_{\text {res }}(H)$ of $U_{\text {res }}(H)$ can be decomposed into a direct sum,

$$
\begin{gathered}
\mathcal{U}_{\mathrm{res}}(H):=\mathfrak{h} \oplus \mathfrak{m}, \quad \mathfrak{h}=\left\{x \mid x_{++}^{*}=-x_{++}, x_{--}^{*}=-x_{--}, x_{+-}=x_{-+}=0\right\}, \\
\mathfrak{m}=\left\{x \mid x_{++}=x_{--}=0, x_{-+}^{*}=-x_{+-}\right\} .
\end{gathered}
$$

As a manifold modelled on its Lie algebra, $U_{\text {res }}(H)$ is a Banach manifold, not a Hilbert manifold. In spite of this fact, we can nevertheless define the same inner product on the subalgebra $V$ of the Lie algebra $\mathcal{U}_{\text {res }}(H)$ consisting of the skewHermitian Hilbert-Schmidt operators as we did for $\mathcal{U}_{H S}(H):\langle x, y\rangle=\operatorname{tr}\left(x^{*} y\right)$.

Moreover, the covariant derivative

$$
\nabla_{x} y:=\frac{1}{2}[x, y], \quad x, y \in \mathcal{U}_{\text {res }}(H),
$$

still makes sense, and when $x, y \in V$ then $\nabla_{x} y \in V$. With respect to this inner product and covariant derivative it also makes sense to speak of a totally geodesic Hilbert submanifold $N$ of $U_{\text {res }}(H)$. One simply requires that geodesics of $N$ are left-translations of 1-parameter groups of the form $\exp (t x), x \in v$. Lastly, we can define as for $U_{H S}(H)$ an involution $\sigma(g):=g^{-1}$. With respect to the inner product on $V, \sigma$ is an isometric involution on $V$. 


\section{Hilbert Grassmannians}

Remark. We use the notation $\operatorname{Gr}(p, q)$ to denote the complex Grassmannian of $p$-planes in $\mathbb{C}^{p+q}$.

Definition 3.1. Let $G r(r, \infty)$ denote the set of complex $r$-planes of $H$. We claim for $r<\infty$ that $\operatorname{Gr}(r, \infty)$ has the structure of a complex Hilbert manifold. It is easy to show that $G L_{H S}(H)$ acts transitively on $G r(r, \infty)$, and hence that $\operatorname{Gr}(r, \infty)$ acquires the structure of a complex Hilbert manifold. Note that $\operatorname{Gr}(r, \infty)$ can also be realized as the coset space $U_{H S}(H) / U(P) \times U_{H S}\left(P^{\perp}\right)$. In this representation $G r(r, \infty)$ inherits the metric of a Riemannian submersion of $U_{H S}(H)$. As in the finite-dimensional case, $G r(r, q), q>r$, the maximal flat torus in $G r(r, \infty)$ has dimension $r$. For this reason we call $G r(r, \infty)$ the rank $r$ Hilbert Grassmannian.

We will now construct the generalization of Cartan's embedding map, $i_{r}: G r(r, \infty) \rightarrow U_{H S}(H)$. Let $N_{r} \subset U_{H S}(H)$ denote the set of unitary operators

$$
\left\{A \mid A^{2}=I, \operatorname{dim}(-1) \text { eigenspace of } A \text { is } r\right\} .
$$

$N_{r}$ has several properties:

1. $N_{r}=g N_{r} g^{*}$ for all $g \in U_{H S}(H)$.

2. Let $\left\{e_{i}\right\}$ denote a complete orthonormal basis for $H$. Let $P=\operatorname{span}\left\{e_{1}, \ldots, e_{r}\right\}$. We define $B:=i_{r}(P) \in U_{H S}(H)$ where $B$ is the unitary operator satisfying $\left.B\right|_{P}=-I$, and $\left.B\right|_{P^{\perp}}=I$. We claim the orbit of $B$ under the smooth action $\tilde{i}_{r}(g):=g B g^{*}, g \in U_{H S}(H)$ is transitive on $N_{r}$. To see this, let $A \in N_{r}$ and let $Q$ denote the -1 eigenspace of $A$. We can construct a unitary operator $g \in U_{H S}(H)$ such that $\tilde{i}_{r}(g)=A$ as follows: Define $\left.g\right|_{P \oplus Q} P=Q$ (in a unitary fashion), and $\left.g\right|_{(P \oplus Q)^{\perp}}=I d$ then $g \in U_{H S}(H)$ and $\tilde{i}_{r}(g)=g B g^{*}=A$.

3. $\tilde{i}_{r}$ is constant on equivalence classes of the form $\left[g\left(U(P) \times U\left(P^{\perp}\right)\right)\right]$. Hence we can define the isometric map $i_{r}: G r(r, \infty) \rightarrow N_{r}$.

4. Since $\sigma\left(g B g^{*}\right)=g B g^{*}, N_{r}$ is invariant under the isometric involution $\sigma$ of $U_{H S}(H)$. Hence $N_{r}$ is a totally geodesic submanifold of $U_{H S}(H)$.

5. A copy $i_{r}(G r(p, q))$ of a compact Grassmannian within $N_{r}$ is characterized by the property $i_{r}(G r(p, q)) \subset U(W) \times I_{W^{\perp}}$ for some $W^{r+q} \subset H$. Conversely, if $W^{r+q} \subset H$ and $Y \subset U(W) \times I_{W^{\perp}} \cap N_{r}$ then there is a compact Grassmannian $G r(r, q) \subset G r(r, \infty)$ and a subset $X \subset G r(r, q)$ with the property $i_{r}(X)=Y$.

From [9] we require one last Hilbert Grassmannian:

Definition 3.2. With respect to the polar decomposition $H=H_{+} \oplus H_{-}$we define $G r(H)$ as the coset space

$$
G r(H):=\frac{U_{\mathrm{res}}(H)}{U\left(H_{+}\right) \times U\left(H_{-}\right)} .
$$

As stated in [9] $\operatorname{Gr}(H)$ is a complex Hilbert manifold with infinite rank modelled on the subspace $\mathfrak{m} \subset \mathcal{U}_{\text {res }}(H)$.

We can also generalize Cartan's embedding map to $G r(H)$. Let $B \in U_{\text {res }}(H)$ denote the unitary operator such that $\left.B\right|_{H_{+}}=I d_{H_{+}},\left.B\right|_{H_{-}}=-I d_{H_{-}}$. Then the map $\tilde{i}_{\infty}: U_{\text {res }}(H) \rightarrow U_{\text {res }}(H)$ given by $\tilde{i}_{\infty}(g)=g B g^{*}$ is a smooth map which is constant on the equivalence classes $\left[g U\left(H_{+}\right) \otimes U\left(H_{-}\right)\right]$. Thus we can define a smooth map $i_{\infty}: G r(H) \rightarrow U_{\text {res }}(H)$. We define $N_{\infty}:=i_{\infty}(G r(H))$. Note that as a manifold, $N_{\infty}$ is a Hilbert manifold contained inside the set $\{B+x \mid x$ HilbertSchmidt\}. Also, $N_{\infty}$ has the same properties as $N_{r}$. 
We recall that a Grassmannian $G r(p, q)$ consists of the set of complex $p$-planes of $\mathbb{C}^{n},(n=p+q)$. $U(n)$ acts in a natural transitive fashion upon $\operatorname{Gr}(p, q)$ by left multiplication. By choosing a distinguished $p$-plane (denoted as $\mathbb{C}^{p}$ ) we can identify $\operatorname{Gr}(p, q)$ with the coset space $U(n) / U\left(\mathbb{C}^{p}\right) \times U\left(\mathbb{C}^{p^{\perp}}\right)=U(n) / U(p) \times U(q)$.

Now for $p \leq r, q<\infty$ there is a (non-canonical) method of totally geodesically embedding $\operatorname{Gr}(p, q)$ within $\operatorname{Gr}(r, \infty)$. One starts first with an $n$-plane $W$ of $H$ and identifies $\mathbb{C}^{n}$ with $W$ by a linear isometry $\phi$. Now take an $(r-p)$ plane $V$ which is orthogonal to $W$. We now construct a map $f: G r(p, q) \rightarrow G r(r, \infty)$ as follows: For every $p$-plane $X$ of $G r(p, q)$ define $f(X)$ to be the $r$-plane $\phi(X) \oplus V$. We state without proof that $f$ is a totally geodesic map. Notice that under Cartan's embedding map $i_{r}$,

- $\left.i_{r} \circ f(X)\right|_{(W \oplus V)^{\perp}}=I_{(W \oplus V)^{\perp}}$ for all $X \in G r(p, q)$.

- The dimension of the (-1) eigenspace of $\left.i_{r} \circ f(X)\right|_{W \oplus V}$ is $r$ while the dimension of the (11) eigenspace of $\left.i_{r} \circ f(x)\right|_{W \oplus V}$ is $q$.

The above can also be applied to embedding copies of $G r(p, q)$ within $G r(H)$ as well. We observe that in our case

1. $V$ is an infinite dimensional subspace of $H$;

2. $V \oplus W$ has an infinite-dimensional complement $U$;

3. there exists an element $g \in U_{\text {res }}(H)$ such that $g^{*} U=H_{+}$and $g^{*}(V \oplus W)=$ $H_{-}$; and

4. we can characterize a copy of $i_{\infty}(G r(p, q))$ with $U_{\text {res }}(H)$ as a set of operators which are constant on a cofinite subspace $X$ of $H$ and whose $(-1)$ eigenspace has dimension $p$ and whose (1) eigenspace has dimension $q$ are in $X^{\perp}$.

We end this section with a last definition:

Definition 3.3. We say a map $f: M \rightarrow G r(r, \infty)$ (resp. $G r(H))$ is full if its image is not contained in some $\operatorname{Gr}(p, q)$ for some $p, q<\infty$.

\section{Properties of $d^{*}$}

Remark. In this section and the following sections $M$ denotes a closed orientable Riemannian manifold unless otherwise stated.

Because we will require the use of $d^{*}$, the formal adjoint of $d$, in our theorem, we will give an explicit computation of the action of $d^{*}$ on local 1-forms in this section.

Let $\left\{\chi_{i}\right\}$ be a local orthonormal basis for $T M$. With respect to $\left\{\chi_{i}\right\}$ we can compute the connection 1-form $\Omega$ of the Levi-Civita connection:

$$
\begin{aligned}
0 & =\chi_{i}\left\langle\chi_{j}, \chi_{k}\right\rangle \\
& =\left\langle\nabla_{\chi_{i}} \chi_{j}, \chi_{k}\right\rangle+\left\langle\chi_{j}, \nabla_{\chi_{i}} \chi_{k}\right\rangle \\
& =\Omega_{i j}^{k}+\Omega_{i k}^{j}, \\
\Omega_{i j}^{k} & =-\Omega_{i k}^{j} .
\end{aligned}
$$

In particular, $\Omega_{i j}^{j}=0$ (no sum). 
Now we consider the exterior differential of $\left\{\chi_{i}^{*}\right\}$, the local dual basis for $T^{*} M$ :

$$
\begin{aligned}
d \chi_{i}^{*}\left(\chi_{j}, \chi_{k}\right) & =\chi_{j} \chi_{i}^{*}\left(\chi_{k}\right)-\chi_{k} \chi_{i}^{*}\left(\chi_{j}\right)-\chi_{i}^{*}\left(\left[\chi_{j}, \chi_{k}\right]\right) \\
& =-\chi_{i}^{*}\left(\sum_{r}\left(\Omega_{j k}^{r}-\Omega_{k j}^{r}\right) \chi_{r}\right) \\
& =-\left(\Omega_{j k}^{i}-\Omega_{k j}^{i}\right) \\
d \chi_{i}^{*} & =-\frac{1}{2} \sum_{j k}\left(\Omega_{j k}^{i}-\Omega_{k j}^{i}\right) \chi_{j}^{*} \wedge \chi_{k}^{*} .
\end{aligned}
$$

And finally, with respect to the local dual basis $\left\{\chi_{i}^{*}\right\}$, the action of $d^{*}$ on a local section $\sum_{i} \alpha_{i} \chi_{i}^{*}$ is given by the formula

$$
\begin{aligned}
d^{*} \sum_{i} \alpha_{i} \chi_{i}^{*} & =-\sum_{i}\left(\chi_{i}\left(\alpha_{i}\right)+\alpha_{i} \sum_{j} \Omega_{j i}^{j}\right) \\
& =-\sum_{i}\left(\chi_{i}\left(\alpha_{i}\right)-\alpha_{i} \sum_{j} \Omega_{j j}^{i}\right) .
\end{aligned}
$$

In the next section we will discuss the behavior of the Laplacian $d^{*} d+d d^{*}$ acting on sections of a Hilbert vector bundle.

\section{Elliptic Operators and Hilbert Vector Bundles}

We will need a finiteness theorem for solutions of a second-order linear elliptic differential equation involving a Hilbert vector bundle. We will discuss here the extensions to Hilbert vector bundles of some results on elliptic operators. For a good introduction to the theory of pseudo-differential operators see [13] or [12].

We know that the Laplacian

$$
\Delta:=d d^{*}+d^{*} d: \Gamma\left(T^{*} M\right) \rightarrow \Gamma\left(T^{*} M\right)
$$

is a second order elliptic operator. The spectrum of $\Delta$ is discrete and unbounded. The kernel of $\Delta$ is the set of closed and co-closed 1-forms of $M$. It is a well-known result of Hodge theory that every cohomology class $[\omega] \in H^{1}(M)$ has a unique representative in the kernel of $\Delta$. Conversely, a basis for the kernel of $\Delta$ is a basis for $H^{1}(M)$. Hence the dimension of the kernel of $\Delta$ is precisely the rank of $H^{1}(M)$. In the case of compact manifolds the rank of $H^{1}(M)$ is finite, hence the kernel of $\Delta$ is finite-dimensional.

We also know that as an operator, $\Delta$, acting on the space $\Gamma\left(T^{*} M\right)$, is diagonalizable. For each eigenvalue $\lambda_{i}$ the dimension of the corresponding eigenspace is finite-dimensional. Let $\left\{v_{i}\right\}$ denote a complete set of orthonormal eigenvectors of $\Delta$ with corresponding eigenvalues $\left\{\lambda_{i} \mid \lambda_{i} \leq \lambda_{i+1}\right\}$. Then $\left\{v_{i}\right\}$ is a formal basis for $\Gamma\left(T^{*} M\right)$. With the exception of the kernel, we can find an inverse for $\Delta$ called the Green's operator and denoted as $G$. As is usually done, we can extend $G$ to the kernel by defining $\left.G\right|_{\text {kernel }} \equiv 0$. Then we see that as defined $G$ is a compact operator acting on $\Gamma\left(T^{*} M\right)$ with kernel the same as the kernel of $\Delta$. It is also a well-known result that the composition of pseudo-differential operators of order 0 or 1 with $G$ are compact operators acting on $\Gamma\left(T^{*} M\right)$.

Similarly, the elliptic operator $\Delta_{0}:=d^{*} d: \mathcal{C}^{\infty}(M) \rightarrow \mathcal{C}^{\infty}(M)$ has a discrete spectrum with a 1-dimensional kernel consisting of the constant functions. Let $\left\{f_{i}\right\}$ 
denote a complete orthonormal basis of eigenvectors for $\mathcal{C}^{\infty}(M)$ with corresponding eigenvalues $\left\{\mu_{i} \mid \mu_{i} \leq \mu_{i+1}\right\}$.

Now we wish to consider the Hilbert vector bundle $V:=T^{*} M \otimes H^{\prime}$ where $H^{\prime}$ denotes the trivial Hilbert vector bundle $M \times H$, with the flat connection $\nabla:=d$.

With the use of a constant orthonormal basis $\left\{e_{i}\right\}$ for $H^{\prime}$ we can extend the action of a pseudo-differential operator $D: \Gamma\left(T^{*} M\right) \rightarrow \Gamma\left(T^{*} M\right)$ as follows: we can decompose a section $\sigma \in \Gamma(V)$ as

$$
\sigma=\sum_{i} \sigma_{i} \otimes e_{i}, \quad \sigma_{i} \in \Gamma\left(T^{*} M\right),
$$

then we define the action of $D$ on $\sigma$ by

$$
D(\sigma):=\sum_{i} D\left(\sigma_{i}\right) \otimes e_{i} .
$$

This extension of pseudo-differential operators of $\Gamma\left(T^{*} M\right)$ is well-defined up to a constant general linear transformation of the basis $\left\{e_{i}\right\}$.

We can also extend the action of an arbitrary smooth linear map $\omega: H^{\prime} \rightarrow H^{\prime}$ to $V$. With respect to the basis of eigenvectors $\left\{f_{i}\right\}, \omega$ can be decomposed (formally) as

$$
\omega:=\sum_{i} f_{i} \otimes \omega_{i}, \quad \omega_{i} \in L(H) .
$$

With respect to this decomposition and the decomposition in equation (26) for a section $\sigma \in \Gamma(V)$ the action of $\omega$ on $\sigma$ is given by

$$
\omega(\sigma):=\sum_{i j} f_{i} \sigma_{j} \otimes \omega_{i}\left(e_{j}\right) .
$$

We mention in passing that this definition should not cause concern about the topological convergence of this sum. We shall be dealing here with smooth functions over a compact domain so there is no question that all of our sums do converge and converge strongly enough that the extension of the pseudo-differential operators from the first term of the tensor product and the linear maps in second term of the tensor product is indeed well-defined.

There is one point however that we do need to stress: In the decomposition of $\omega$ in equation (28) the most that we can assume in general about $\omega_{i}$ is that it is a bounded linear operator acting on the Hilbert space $H$. So the composition of the extension of the Green's operator $G$ with the extension of $\omega$ is in general not a Hilbert-Schmidt operator acting on $\Gamma(V)$. Fortunately, in our case we will be looking at an $\omega$ which is the differential of a map $f: M \rightarrow U_{H S}(H)$. Since $\mathcal{U}_{H S}(H)$ is a Hilbert-Schmidt Lie algebra, $\omega_{i}$ will necessarily be a Hilbert-Schmidt operator. The proof is simple to state. To determine $\omega_{i}$ one simply does as in Fourier analysis when one is determining the decomposition of a continuous function $f: S^{1} \rightarrow S^{1}$ into a series $a_{n} e^{i n \theta}$. We integrate $\omega * f_{i}$ over $M$ using the volume form $d V$ of $M$ to determine $\omega_{i}$. Since $M$ is compact it is easy to see that

$$
\sum_{j}\left\|\omega_{i}\left(e_{j}\right)\right\|^{2}=\int_{M} \sum_{j}\left\|f_{i} \omega\left(e_{j}\right)\right\|^{2} d V \leq \int_{M}\|\omega\|^{2} d V<\infty
$$

and thus $\omega_{i}$ is a Hilbert-Schmidt operator. 
In Section [7] we will investigate the space of solutions of a second-order elliptic differential equation of the form

$$
\Delta \tau+d \nu \tau \equiv 0
$$

where $\tau$ is a section of $V$, and $\nu: \Gamma(V) \rightarrow \Gamma(H)$ is a 0th order pseudo-differential operator, and in the decomposition of $\nu$ into its components $\nu:=\sum_{i} f_{i} \otimes \nu_{i}, \nu_{i}$ is a compact operator. We require for our proofs that the space of solutions of this equation be finite-dimensional. Ideally, we would like to apply $G$ to both sides in order to solve for $\tau$. However, if $\Delta$ has a non-zero kernel, then the composition $G \circ \Delta \neq I$ except for those sections which are orthogonal to the kernel of $\Delta$. The standard solution to this problem would be to add to $\Delta$ and $G$ the 0th order projection operator $I_{0}$ that is the identity operator on the kernel of $\Delta$ and zero on the orthogonal complement of the kernel of $\Delta$. We can extend $I_{0}$ to $I_{0} \otimes I$ to act on the sections of $V$. The new differential equation would be

$$
\left(\Delta+I_{0}\right) \tau+\left(d \nu-I_{0}\right) \tau \equiv 0 .
$$

We could now "solve" this equation by applying $G+I_{0}$ to both sides:

$$
\begin{aligned}
& 0 \equiv\left(G+I_{0} \otimes I\right)\left(\left(\Delta+I_{0}\right) \tau+\left(d \nu-I_{0}\right) \tau\right), \\
& 0 \equiv \tau+\left(G+I_{0}\right)\left(d \nu-I_{0}\right) \tau .
\end{aligned}
$$

If $V$ were a finite-dimensional vector bundle the extension of $I_{0}$ to $I_{0} \otimes I$ would be a compact operator. Hence $\left(G+I_{0}\right) d\left(\nu-I_{0}\right)$ would be a compact operator. And therefore the space of solutions of equation (34) would be finite-dimensional by the Fredholm alternative.

Unfortunately in the case of the Hilbert bundle $V$ the extension of the operator $I_{0}$ to $I_{0} \otimes I$ is not compact. We cannot automatically conclude $\left(G+I_{0}\right)\left(d \nu-I_{0}\right)$ is compact. Thus we cannot conclude that the space of solutions is automatically finite-dimensional.

There are two solutions to this dilemma. The first solution is to assume the kernel of $\Delta$ is zero, in other words that $H^{1}(M)=0$. In this case we do not require perturbing $\Delta$ and $G$. We only assume that in the decomposition of $\nu$ all of the $\nu_{i}$ components be compact. The second solution is by assuming the extra condition that $\tau$ and $d \nu \tau$ are orthogonal to the kernel of $\Delta$ in equation (31). In our proof in the next section we will in fact use the second solution.

\section{HARMONIC MAPS}

Definition 6.1. A map $f: M \rightarrow N$ is said to be a harmonic map if it is a critical point of the action

$$
\mathcal{A}(f):=\int_{M}\|d f\|^{2} d V_{M}
$$

where $\|d f\|$ is the usual Hilbert-Schmidt norm with respect to the Riemannian metrics of $M$ and $N$. It is a standard exercise to show that the Euler-Lagrange equation for this action is given by the formula

$$
\operatorname{tr} \nabla d f \equiv 0 .
$$

The expression $\operatorname{tr} \nabla d f$ is sometimes referred to as the tension field of $f$. Here $\nabla$ denotes the covariant derivative of $T^{*} M \otimes f^{-1} T N$ induced by the covariant 
derivatives of $T^{*} M$ and $f^{-1} T N$. $\operatorname{tr} \nabla d f$ is computed in a local orthonormal basis $\left\{\chi_{i}\right\}$ for $T M$ as

$$
\operatorname{tr} \nabla d f:=\sum_{i} \nabla_{\chi_{i}}(d f)\left(\chi_{i}\right)
$$

With the use of $\left\{\chi_{i}\right\}$, a dual basis $\left\{\chi_{i}^{*}\right\}$ for $T^{*} M$, and an orthonormal basis $\left\{\zeta_{j}\right\} \subset \mathcal{U}_{H S}(H)$ we can decompose $d f$ as

$$
d f:=\omega \equiv \sum_{i j} \omega_{i j} \chi_{i}^{*} \otimes \zeta_{j}, \quad \omega_{i j} \in \mathcal{C}^{\infty}(G)
$$

We take advantage of this decomposition to rewrite and simplify the tension field equation for $f$.

$$
0 \equiv \operatorname{tr} \nabla d f=\sum_{i} \nabla_{\chi_{i}}(d f)\left(\chi_{i}\right)=-d^{*} \omega
$$

So now we see that a map $f: M \rightarrow U_{H S}(H)$ is harmonic iff the differential $\omega:=d f$ has divergence zero. As the differential of a map $f: M \rightarrow U_{H S}(H), \omega$ can also be viewed as a flat connection 1 -form with values in $\mathcal{U}_{H S}(H)$. Hence $\omega$ must satisfy the non-linear differential equation

$$
d \omega+\omega \wedge \omega \equiv 0
$$

We mention two further crucial properties of harmonic maps:

1. If $\pi: L \rightarrow M$ is an isometric covering map and $f: M \rightarrow N$ is a harmonic map, then $f \circ \pi: L \rightarrow N$ is a harmonic map as well.

2. If $L$ is a totally geodesic submanifold of $N$ and $f: M \rightarrow L$ is a harmonic map, then $i \circ f: M \rightarrow N$ is a harmonic map as well. Here $i: L \hookrightarrow N$ is the inclusion map.

\section{The Theorems}

Definition 7.1. We say a map $f: M \rightarrow U_{H S}(H)$ (resp. $U_{\text {res }}(H)$ ) has image contained in a finite-dimensional subgroup $U(n)$ of $U_{H S}(H)$ (resp. $\left.U_{\text {res }}(H)\right)$ if there exists an $n$ dimensional vector space $W \subset H$ such that $\left.f(x)\right|_{W^{\perp}} \equiv I_{W^{\perp}}$ (for all $x \in M$ ), or in other words, the operator $f(x)$ is trivial on $W^{\perp}$ for all $x$ in $M$. Equivalently, if

$$
\left.d f(\chi)\right|_{W^{\perp}}=\left.\omega(\chi)\right|_{W^{\perp}} \equiv 0, \quad \forall \chi \in T M .
$$

Without any further preliminaries here is the main theorem:

Theorem 1. If $M$ is a closed Riemannian manifold, then every harmonic map $f: M \rightarrow U_{H S}(H)$ with $f(p)=I d$ for some $p \in M$ has image contained inside a finite-dimensional subgroup $U(n)$ of $U_{H S}(H)$.

Proof. Let $f: M \rightarrow U_{H S}(H)$ be a harmonic map. Without loss of generality we can assume $M$ is orientable, since if it is not then the double cover $L$ of $M, \pi: L \rightarrow M$, is an orientable compact manifold. Thus the map $f \circ \pi: L \rightarrow U_{H S}(H)$ is a harmonic map with the same image as $f(M)$. We will prove that

$$
\left.d f(\chi)\right|_{W^{ \pm}}=\left.\omega(\chi)\right|_{W^{\perp}} \equiv 0, \quad \forall \chi \in T M
$$

for some $W^{\perp}$.

Our proof has two steps. First we derive a second order linear elliptic equation. We choose a fixed orthonormal basis $\left\{e_{i}\right\}$ for the Hilbert space $H$ which $U_{H S}(H)$ 
acts. We define an orthonormal basis for sections of the Hilbert bundle $M \times H$ by defining

$$
\sigma_{i}(x):=(f(x))^{-1} e_{i}, \quad x \in M,
$$

where $(f(x))^{-1}$ denotes the group inversion of the element of $f(x)$ in $U_{H S}(H)$. It follows that $\sigma_{i}$ satisfies the differential equation

$$
d \sigma_{i}+\omega \sigma_{i} \equiv 0 .
$$

By composing equation (44) with $d^{*}$ we get a second-order elliptic equation in $\sigma_{i}$ :

$$
\Delta_{0} \sigma_{i}+d^{*}\left(\omega \sigma_{i}\right) \equiv 0 .
$$

which can be simplified further:

$$
\begin{aligned}
0 & =\Delta_{0} \sigma_{i}+d^{*}\left(\omega \sigma_{i}\right) \\
& =\Delta_{0} \sigma_{i}-\sum_{k j}\left(\chi_{k} \omega_{k j}\right) \zeta_{j}\left(\sigma_{i}\right)-\sum_{k j l} \omega_{k j} \zeta_{j}\left(\chi_{k} \sigma_{i}\right) \\
& =\Delta_{0} \sigma_{i}-0-\sum_{k j l} \omega_{k j} \zeta_{j}\left(\chi_{k} \sigma_{i}\right) \quad\left(d^{*} \omega=0\right), \\
0 & =\Delta_{0} \sigma_{i}-\left\langle\omega, d \sigma_{i}\right\rangle,
\end{aligned}
$$

where $\left\langle\omega, d \sigma_{i}\right\rangle$ is the $\mathbb{C}$-bilinear extension of the inner product $\langle\rangle:, T^{*} M \times T^{*} M \rightarrow$ $\mathcal{C}^{\infty}(M)$ to vector and linear operator sections of $V$ and $T^{*} M \otimes L(H)$ respectively.

Unfortunately, the kernel of $\Delta_{0}$ is the set of constant sections of $M \times H$ and we cannot guarantee that $\sigma_{i}$ is orthogonal to the kernel of $\Delta_{0}$. However, by taking a further exterior differential we have

$$
\begin{aligned}
& 0=d \Delta_{0} \sigma_{i}-d\left\langle\omega, d \sigma_{i}\right\rangle, \\
& 0=d d^{*}\left(d \sigma_{i}\right)-d\left\langle\omega, d \sigma_{i}\right\rangle .
\end{aligned}
$$

Since $d^{*} d\left(d \sigma_{i}\right)=0$ we can add in $0=d^{*} d\left(d \sigma_{i}\right)$ to equation (51) and arrive at a second-order linear elliptic equation in $d \sigma_{i}$ :

$$
0=\Delta\left(d \sigma_{i}\right)-d\left\langle\omega, d \sigma_{i}\right\rangle .
$$

We claim now that $d \sigma_{i}$ is orthogonal to the kernel of $\Delta$. Let $\alpha \in \operatorname{ker} \Delta=$ $H^{1}(M) \otimes H$. Then we have

$$
\int_{M}\left\langle d \sigma_{i}, \alpha\right\rangle d V=\int_{M}\left\langle\sigma_{i}, d^{*} \alpha\right\rangle d V=0 \text { since } \alpha \text { is coclosed. }
$$

By an identical proof $d\left\langle\omega, d \sigma_{i}\right\rangle$ is also orthogonal to the kernel of $\Delta$. Thus we can invert our equation using the Green's operator $G$ :

$$
\begin{aligned}
\Delta\left(d \sigma_{i}\right)-d\left\langle\omega, d \sigma_{i}\right\rangle & =0, \\
G \Delta\left(d \sigma_{i}\right)-G d\left\langle\omega, d \sigma_{i}\right\rangle & =0, \\
I\left(d \sigma_{i}\right)-G d\left\langle\omega, d \sigma_{i}\right\rangle & =0 .
\end{aligned}
$$

From Section 5 we know $G d\left\langle\omega,_{-}\right\rangle$is a compact operator on the space of sections of $V$ orthogonal to ker $\Delta$. Thus equation (56) has only a finite-dimensional space of solutions.

Now for our second step in the proof we will determine a $W^{\perp} \subset H$ for which $\left.\omega(x)\right|_{W^{\perp}} \equiv 0$, for all $x \in M$.

Without loss of generality we assume that $\left\{d \sigma_{1}, \ldots, d \sigma_{n}\right\}$ is a basis for the span of $\left\{d \sigma_{i}\right\}_{i=1}^{\infty}$. 
For $j>n, d \sigma_{j}$ is linearly dependent on $\left\{d \sigma_{1}, \ldots, d \sigma_{n}\right\}$, so we can write

$$
d \sigma_{j}=\sum_{i=1}^{n} \alpha_{j i} d \sigma_{i}, \quad \text { all } \alpha_{j i} \text { constant. }
$$

Hence

$$
0=d\left(\sum_{i=1}^{n} \alpha_{j i} \sigma_{i}-\sigma_{j}\right)
$$

and so, $\eta_{j}:=\sum_{i=1}^{n} \alpha_{i j} \sigma_{i}-\sigma_{j}$ is a constant section of $M \times H$. $\eta_{j}$ satisfies the following two properties:

1. $\omega \eta_{j} \equiv 0$.

Proof.

$$
\begin{aligned}
\omega \eta_{j} & =\omega\left(\sum_{i=1}^{n} \alpha_{i j} \sigma_{i}-\sigma_{j}\right) \\
& =\sum_{i=1}^{n} \alpha_{i j} \omega \sigma_{i}-\omega \sigma_{j} \\
& =-\left(\sum_{i=1}^{n} \alpha_{i j} d \sigma_{i}-d \sigma_{j}\right) \\
& =-d\left(\sum_{i j}^{n} \alpha_{i j} \sigma_{i}-\sigma_{j}\right) \\
& =0 .
\end{aligned}
$$

(64) the span of $\left\{\sigma_{i}(x)\right\}_{i=1}^{n} \cup\left\{\eta_{j}\right\}=$ the span of $\left\{\sigma_{i}(x)\right\}_{i=1}^{n} \cup\left\{\sigma_{j}\right\} \quad(\forall x \in M)$

Proof. Since $\eta_{j}=\sum_{i=1}^{n} \alpha_{i j} \sigma_{i}-\sigma_{j}$ and $\sigma_{j}=\sum_{i=1}^{n} \alpha_{i j} \sigma_{i}-\eta_{j}$. Let

$$
W^{\perp}:=\overline{\operatorname{span}\left\{\eta_{j}\right\}_{j=n+1}^{\infty}} \text {. }
$$

Then it is clear since

$$
\operatorname{span}\left\{\sigma_{i}(x)\right\}_{i=1}^{n} \oplus W^{\perp}=\overline{\operatorname{span}\left\{\sigma_{i}(x)\right\}_{i=1}^{n} \cup\left\{\sigma_{j}(x)\right\}_{j=n_{1}}^{\infty}}=H
$$

for all $x \in M$ that $W:=W^{\perp \perp}$ has to be of dimension $n$. $\left.\omega\right|_{W^{\perp}}=0$ implies $\left.f(x)\right|_{W^{\perp}}$ is constant. Since $\left.f(p)\right|_{W^{\perp}}=I_{W^{\perp}}$ then $\left.f(x)\right|_{W^{\perp}}=I_{W^{\perp}}$. Since $f$ is a unitary operator, $f$ has to have image contained in $U(n) \cong U(W) \times I_{W^{\perp}}$.

Remark. The proof of the theorem is the same as in [7].

Remark. The theorem is true as well if we replace $U_{H S}(H)$ with $U_{\text {res }}(H)$. The only additional hypothesis that we require is that $d f$ should be Hilbert-Schmidt valued. In the case of maps $f: M \rightarrow G r(H)$ composed with $i_{\infty}, d\left(i_{\infty} \circ f\right)$ is trivially Hilbert-Schmidt valued. Since left-multiplication by an operator $g \in U_{\text {res }}(H)$ is an isometry, then the map $h(x)=\left(i_{\infty} \circ f(p)\right)^{-1} i_{\infty} \circ f(x)$ satisfies the hypothesis of the theorem.

Corollary 2. If $M$ is a closed Riemannian manifold and $f: M \rightarrow G r(r, \infty)$ is a harmonic map, then $f$ cannot be full. 
Proof. By investigating the properties of $i_{r} \circ f: M \rightarrow U_{H S}(H)$ we will derive sufficient information of the map $f: M \rightarrow U_{H S}(H)$ to find a compact Grassmannian which contains $f(M)$.

We begin with: let $g:=i_{r} \circ f(p)$ for some $p \in M$. Let $H_{r}$ denote the $(-1)$ eigenspace of $g$. Let $H_{\infty}$ denote the (1) eigenspace of $g$. From the previous theorem we know $g^{*} i_{r} \circ f$ has image in some $U(V) \times I_{V \perp}, s:=\operatorname{dim}(V)<\infty$. Hence $i_{r} \circ f \subset g\left(U(V) \times I_{V^{\perp}}\right)$.

Let $W:=V+H_{r}$. We claim now that $g\left(U(V) \times I_{V^{\perp}}\right) \subset U(W) \times I_{W^{\perp}}$.

Proof.

$$
\begin{aligned}
x \in W^{\perp} & \Rightarrow x \in V^{\perp} \cap H_{r}^{\perp}, \\
& \Rightarrow \forall A \in U(V) \times I_{V^{\perp}}, \quad g A x=g x=x, \\
& \Rightarrow \forall A \in U(V) \times I_{V^{\perp}}, \quad g A \in U(W) \times I_{W^{\perp}}, \\
& \Rightarrow g\left(U(V) \times I_{V^{\perp}}\right) \subset U(W) \times I_{W^{\perp}} .
\end{aligned}
$$

Since $i_{r} \circ f(M) \subset g\left(U(V) \times I_{V^{\perp}}\right)$ then $i_{r} \circ f(M) \subset U(W) \times I_{W^{\perp}}$. This implies that $f(M) \subset G r(r, s-r)$ for some compact Grassmannian $G r(r, s-r)$ from property 5 of Cartan's embedding map. Thus $f$ cannot be full.

Corollary 3. If $M$ is a closed Riemannian manifold and $f: M \rightarrow G r(H)$ is a harmonic map, then $f$ cannot be full.

Proof. The proof is essentially the same as in the previous corollary. The only difference is that the dimensionality $p$ of the $(-1)$ eigenspace of $i_{\infty} \circ f(x)$ will not be the same for all $x \in M$. However, $p$ will be bounded by $t:=\operatorname{dim} W$. This will allow us to conclude that $f(M)$ is contained in a finite union of compact Grassmannians $\{G r(p, t-p) \mid p=0,1, \ldots, t\}$. It is always possible to find a larger compact Grassmannian $\operatorname{Gr}\left(p^{\prime}, q^{\prime}\right)$ which contains a finite collection of compact Grassmannians. Hence $f$ cannot be full.

\section{Conclusions}

The proof of the main theorem of this paper was the same as in [7]. In both cases, we could associate a connection $\omega$ with the harmonic map $f$, a connection whose divergence vanished. It was the realization that the divergence of $\omega$ did not depend on the topology of $M$ that allowed us to extend the theorem to all closed Riemannian manifolds. Once we knew that the theorem extended to all closed Riemannian manifolds it was easy to extend the finiteness result to maps into Hilbert Grassmannians by taking advantage of the Cartan embedding map, and the behavior of harmonic maps under composition with totally geodesic maps.

The results of this paper lead to an interesting speculation in physics: If one were to assume that spacetime were compact and that the fields observed in nature arise as critical points of a harmonic-like action into a Hilbert Lie group then it is natural to expect the gauge groups of all the forces in nature to be finite-dimensional. By determining the energies of the actions of the observed forces in nature correlated with the homotopy classes of maps from spacetime into a Hilbert Lie group, we could conceivably determine the topology of spacetime. In my next paper, I intend to investigate the relationship between Yang-Mills instantons and harmonic maps as it applies to physical phenomena. 
In conclusion, this paper is an extension of the results obtained by the author in connection with the properties of harmonic maps from compact Lie groups into a Hilbert orthogonal group.

\section{REFERENCES}

1. M. F. Atiyah and D. W. Anderson, K-Theory, Harvard University, 1964. MR 90m:18011

2. Theodor Bröcker and Tammo tom Dieck, Representations of Compact Lie Groups, SpringerVerlag, 1985.

3. D. Burns, Harmonic Maps from $\mathbb{C} P^{1}$ to $\mathbb{C} P^{n}$, Proc. Conf. on Harmonic Maps, SpringerVerlag, p. 217-263, 1980.

4. J. Eells and J. C. Wood, Harmonic Maps from Surfaces to Complex Projective Spaces, Advances in Math. (1983), no. 49. MR 85f:58029

5. James Eells and Andrea Ratto, Harmonic Maps and Minimal Immersions with Symmetries, Methods of Ordinary Differential Equations Applied to Elliptic Variational Problems, Anals of Mathematics Studies, no. 130, Princeton University Press, Princeton, New Jersey, 1993. MR 94k:58033

6. D. S. Freed, Flag Manifolds and Infinite Dimensional Kähler Geometry, Infinite Dimensional Groups with Applications, Publ. Math. Sci. Res. Inst. 4, p. 83-124, 1985. MR 87k:58020

7. Rodrigo P. Gomez, A finiteness theorem of harmonic maps from compact Lie groups to $O_{H S}(H)$, Manuscripta Mathematica, no. 93, p. 325-335, 1997. MR 98d:58043

8. S. Kobayashi and K. Nomizu, Foundations of Differential Geometry, vol. I, II, Wiley, 1963, 1969. MR 97c:53001a MR 97c:53001b

9. Andrew Pressley and Graeme Segal, Loop Groups, Oxford University Press, New York, 1988. MR 88i:22049

10. Graeme Segal, Loop Groups and Harmonic Maps, London Math. Soc. Lecture Note Ser. 139, Cambridge Univ. Press, Cambridge, p. 153-164, 1989. MR 91m:58043

11. Karen Uhlenbeck, Harmonic Maps into Lie Groups (Classical solutions of the Chiral Model), J. Differential Geom. 30, no. 1, p. 1-50, 1989. MR 90g:58028

12. Frank W. Warner, Foundations of Differentiable Manifolds and Lie Groups, Springer-Verlag, 1987. MR 84k:58001

13. R. O. Wells, Differential Analysis on Complex Manifolds, Springer-Verlag, 1980. MR 83f:58001

Comprehensive Studies Program, University of Michigan, Ann Arbor, Michigan 48109

Current address: 8838 Tides Ebb Ct., Columbia, Maryland 21045

E-mail address: rpgomez@yahoo.com 